\title{
Dictynna
}

Dictynna

Revue de poétique latine

15 | 2018

Varia

\section{Rex genitor: Creonte, Edipo e la tragedia del potere nella Tebaide di Stazio}

\section{Stefano Briguglio}

\section{(2) OpenEdition}

\section{Journals}

\section{Edizione digitale}

URL: http://journals.openedition.org/dictynna/1641

DOI: 10.4000/dictynna.1641

ISSN: 1765-3142

\section{Notizia bibliografica digitale}

Stefano Briguglio, «Rex genitor: Creonte, Edipo e la tragedia del potere nella Tebaide di Stazio », Dictynna [En ligne], 15 | 2018, mis en ligne le 20 décembre 2018, consulté le 10 septembre 2020. URL : http://journals.openedition.org/dictynna/1641 ; DOI : https://doi.org/10.4000/dictynna.1641

Questo documento è stato generato automaticamente il 10 settembre 2020

\section{(c) (i) (9)}

Les contenus des la revue Dictynna sont mis à disposition selon les termes de la Licence Creative Commons Attribution - Pas d'Utilisation Commerciale - Pas de Modification 4.0 International. 


\title{
Rex genitor: Creonte, Edipo e la tragedia del potere nella Tebaide di Stazio
}

\author{
Stefano Briguglio
}

\section{NOTE DELL'AUTORE}

Ringrazio gli anonimi referee della rivista, i cui suggerimenti mi hanno permesso di sviluppare alcuni punti del lavoro.

1 Libro undicesimo della Tebaide. Dopo lo scontro fratricida è Creonte a prendere il potere a Tebe, ma la maledizione che fin dal massacro degli Sparti grava sulla città non si estingue con la morte dei due fratelli nemici. Ogni sovrano, appena salito al trono, è destinato a trasformarsi in un tiranno, come se Tebe non potesse essere retta altrimenti': potere politico e autocrazia nel poema coincidono. Così è per Edipo (già nella tradizione greca), così per i suoi figli e così per Creonte, la cui progressiva trasformazione in tiranno è esplorata da Stazio con strumenti sofisticati, in cui la memoria letteraria è messa al servizio di un'indagine psicologica più approfondita di quella delle tragedie attiche di argomento tebano.

2 Si può iniziare dal lessico. La ciclicità ossessiva è una delle cifre che caratterizzano il poema di Stazio ${ }^{2}$ : il passato e il presente si intrecciano e interferiscono senza sosta, in un incubo senza apparente via d'uscita. Anche il potere politico a Tebe è sottoposto a questa legge: come nel primo libro del poema l'azione aveva preso avvio con la metamorfosi in tiranno di Eteocle, così, una volta salito al trono Creonte, la maledizione dell'autocrazia ricompare. Per far risaltare questo tessuto connettivo che collega, anche a distanza, personaggi ed episodi del poema, Stazio ricorre alla ripetizione puntuale di immagini e di lessico 'tematico'.

3 Non è un caso che l'inizio della tirannide di Creonte sia raffigurato esattamente come l'inizio della tirannide di Eteocle. Se è vero che fra l'undicesimo e il dodicesimo libro 
l'epica di Stazio 'si spezza's, è altrettanto vero che proprio quando il lettore si attenderebbe la conclusione della vicenda tragica (con la morte dei due fratelli), la storia sembra pericolosamente destinata a ripetersi ${ }^{4}$. Mentre il lettore si interroga sul futuro di Tebe, il suo sguardo è ancora una volta costretto a rivolgersi al passato, a ritornare al primo libro del poema: leggiamo il testo (Theb. 11, 648-58):

Et iam laeta ducum spes elusisse duorum

res Amphionias alio sceptrumque maligna transtulerat Fortuna manu, Cadmique tenebat iura Creon. Miser heu bellorum terminus! Illi pugnarant fratres. Hunc et Mavortia clamant semina, et impensus patriae paulo ante Menoeceus conciliat populis. Scandit fatale tyrannis flebilis Aoniae solium: pro blanda potestas et sceptri malesuadus amor! Numquamne priorum haerebunt documenta novis? Iuvat ecce nefasto stare loco regimenque manu tractare cruentum.

Ma già la Sorte, lieta d'essersi fatta gioco delle speranze dei due condottieri, aveva con mano perversa tramandato ad altri il regno e lo scettro di Anfione: Creonte teneva il potere di Cadmo. Ah, miserevole conclusione della guerra! È per lui che hanno combattuto i fratelli. Ed è lui che acclamano i discendenti del drago di Marte; e la morte di Meneceo, poco prima sacrificato alla patria, gli attira il favore del popolo. Sale piangendo sul trono fatale ai tiranni della Beozia. Ah seduzione del potere, e amore dello scettro, che è così malvagio consigliere! Dunque gli esempi dei predecessori non saranno mai ricordati dai nuovi venuti? Guarda! Creonte è felice di starsene su un soglio maledetto e tenersi stretta la sanguinosa tirannide ${ }^{5}$.

Così è descritto l'esordio di Creonte al potere: ma questi versi vanno confrontati col passo dell'inizio della Tebaide in cui Stazio narra l'ascesa di Eteocle al trono, successiva al sorteggio tra i due fratelli. Anche in Theb. 1, 139-41 (sic iure maligno / fortunam transire iubent, ut sceptra tenentem / foedere praecipiti semper novus angeret heres 'arrogandosi così un diritto perverso, imposero alla fortuna i suoi mutamenti, in modo che chi impugnava lo scettro, per l'incombente scadenza del patto, fosse sempre angosciato dall'idea del successore futuro') e 175-6 (partiti versant populorum fata manuque / fortunam fecere levem 'si sono spartiti i destini della gente e li manovrano a loro piacimento, e così facendo pretendono di indurre la fortuna a cambiare continuamente') la Fortuna era stata personificata, ma con una differenza significativa: lì era l'oggetto su cui Eteocle e Polinice esercitavano la propria violenza, qui è invece la protagonista che, lieta di aver frustrato le speranze dei due, passa lo scettro a Creonte. Le immagini sono uguali e opposte, identico è il lessico, come dimostra la presenza di malignus $(1,139 \sim 11,649)$, manus $(1,175 \sim 11,650)$, ius $(1,139 \sim 651)$. Ma non basta: il trono di Tebe è fatale per chi vi siede (11, 654-5): per rimarcare questa verità Stazio ricorre ancora a un'immagine (e a un riecheggiamento metrico-sintattico) del primo libro, questa volta dal proemio $(1,34)$ : geminis sceptrum exitiale tyrannis ('lo scettro che fu fatale a due tiranni').

Nonostante la lezione che dovrebbe aver imparato, però, Creonte si compiace di sedere su un trono maledetto (11, 658-9 iuvat ecce nefasto / stare loco, regimenque manu tractare cruentum) $)^{6}$ in questo caso il dialogo con il primo libro diventa ancora più fitto. In Theb. $1,155 \mathrm{ss} .$, il narratore aveva riferito il paradosso alla base dello scontro fra Eteocle e Polinice, una guerra fratricida per un regno povero ${ }^{7}$, e aveva infine commentato (vv. 162-4): loca dira arcesque nefandae ${ }^{8}$ / suffecere odio, furiisque inmanibus emptum / Oedipodae 
sedisse loco 'ma bastano quei luoghi maledetti e una cittadella innominabile a scatenare l'odio: con inaudita follia è stato conquistato il privilegio di sedere sul trono di Edipo'. Proprio come i due fratelli, così anche Creonte, accecato dal potere, non si rende conto della maledizione che il trono di Tebe porta con sé: la storia si ripete.

7 La considerazione del narratore a questo proposito è lapidaria, espressa sotto forma di una interrogativa retorica (11, 656-7): numquamne priorum / haerebunt documenta novis? Il tono e il lessico della domanda la accostano più al genere storiografico che all'epica, come è stato visto"; ma essa serve, una volta di più, a siglare il definitivo ritorno al passato $^{10}$. La lezione della storia è destinata a restare ignorata, quando sono in gioco interessi di potere.

Creonte, è stato detto, è un tiranno da manuale ${ }^{11}$. La sua metamorfosi lo porta nel giro di pochi versi ad assumerne tutte le caratteristiche, fino al gesto di efferatezza estrema, il divieto, cioè, di seppellire i corpi dei caduti.

9 La tradizione filosofica e letteraria antica, è noto, aveva riflettuto sulle caratteristiche del tiranno almeno fin dai poeti tragici, da Platone (Repubblica) e da Senofonte (Ierone): nella cultura romana il discorso era stato approfondito e specializzato in numerosi testi, principalmente epici e tragici ${ }^{12}$. Erede di importanti riflessioni sul potere assoluto, Stazio impara a indagare l'animo del tiranno soprattutto grazie al teatro senecano: in particolar modo, è l'amore totalizzante per il potere - importante lascito della tragedia di Seneca ${ }^{13}$ - a dominare i re di Tebe e a pervertirne la natura, asservendola ${ }^{14}$.

Creonte non fa eccezione: fin dai primi versi che ne descrivono l'ascesa al trono, Stazio pone l'accento sul rapporto erotico tra questi e il potere. Un rapporto esclusivo, forse ancor più devastante di quello che i sovrani di Seneca nutrivano per il trono, poiché annulla ogni sentimento paterno in Creonte. Ma andiamo con ordine.

11 Rileggiamo i vv. 655-6 del libro 11: pro blanda potestas / et sceptri malesuadus amor! Il commento del narratore interpreta i pensieri di Creonte; il potere è dolce e seducente $(\text { blanda })^{15}$ - come già lo aveva definito Tideo in Theb. 2, 399 dulcis amor regni blandumque potestas $^{16}$ 'è cosa dolce la passione del regno e seducente il potere'. In molti luoghi del poema Stazio aveva indagato sulla passione per il trono che animava Eteocle e Polinice ${ }^{17}$ : ora, alla loro morte, lo stesso sentimento si fa strada in Creonte. Nei figli di Edipo la passione politica snaturava il legame tra due fratelli, portandoli alla contesa; in Creonte, questo amore ne rimpiazza un altro, che secondo natura dovrebbe essere più forte: quello per Meneceo. Grazie al suicidio del figlio Creonte ottiene il trono, come si legge in Theb. $11,653^{18} \mathrm{e}$ come riconosce il padre durante il suo lamento funebre. Nel nuovo sovrano, dunque, confliggono due sentimenti contrapposti, quello di re e quello di padre, come nota Stazio con grande finezza psicologica quando definisce Creonte rex genitor (Theb. 12, 61) - un nesso che diventa quasi un ossimoro ${ }^{19}$.

L'intreccio dei due sentimenti è espresso con grande efficacia: il suicidio del giovane guadagna al padre il favore popolare (Theb. 11, 653-5), ma subito la passione per il trono fa sì che Creonte desideri un regno maledetto, che venga privato della sua natura di padre e dimentichi Meneceo (Theb. 11, 659-60) ${ }^{20}$ :

Quid, melior fortuna, potes! Iam flectere patrem

incipit $^{21}$ atque datis abolere Menoecea regnis.

Che grande potere hai sugli uomini, o Fortuna, e quanto li favorisci! Ha appena ricevuto il regno che già comincia a mutare il suo cuore di padre e a scordarsi di Meneceo. 
Senza mezzi termini, Stazio afferma che il regnum porta Creonte a mutare il suo animo di padre (flectere patrem) e a dimenticare Meneceo: lo rimarca l'uso del concreto per l'astratto (patrem) e l'iperbato del nesso datis... regnis (la causa) che incornicia abolere Menoecea (l'effetto). Una passione, dunque, ha preso il posto di un'altra: già Paola Venini aveva rapidamente notato nel suo commento ${ }^{22}$ che il v. 660 allude a Verg. Aen. 1, 719-22. Cupido, su ordine della madre Venere, siede in braccio a Didone e cancella dall'animo della regina l'amore per il defunto Sicheo:

At memor ille

matris Acidaliae paulatim abolere Sychaeum

incipit et vivo temptat praevertere amore

iam pridem resides animos desuetaque corda.

... e quello, memore della madre acidalia, a poco a poco inizia a cancellare Sicheo, e tenta di vincere con un vivo amore l'animo di lei, già da tempo intorpidito, e il suo cuore non più abituato.

Il verbo adoperato e l'azione (registrata agli inizi, e come incipit in entrambi i testi) sono gli stessi; un amore ne scaccia un altro: Enea prende il posto di Sicheo, il trono tebano sostituisce Meneceo.

Ma il paradosso è tale che anche durante il compianto sul corpo del figlio Creonte arriva a dimenticarlo: in Theb. 12, 60-103 Stazio, con un autentico tour de force, costruisce un lamento funebre il cui protagonista non è il defunto, ma colui che lo pronuncia, e in cui, ancora una volta, è il potere assoluto il pensiero dominante ${ }^{23}$.

Iniziamo dal confronto con il modello greco, le Fenicie di Euripide. Stazio segue da vicino la tragedia: più che le affinità, però, sono le differenze con il modello ad acquistare importanza. Nelle Fenicie, Creonte leva il lamento funebre per Meneceo prima del duello fratricida, quando ancora lo scettro di Tebe non è passato nelle sue mani (vv. 1310-21); solo a partire dai vv. 1584ss., dunque alla fine della tragedia, il nuovo sovrano rivelerà la propria natura tirannica, come voleva la tradizione letteraria (almeno fin dall'Antigone di Sofocle). L'amore per Meneceo e quello per il potere non si scontrano, nel Creonte di Euripide: il padre, non ancora re, lascia libero sfogo al suo dolore, presentandosi come un vecchio senza forze che cerca l'aiuto della sorella Giocasta (vv. 1317-9) ${ }^{24}$ :

$\dot{\varepsilon} \gamma \grave{\omega} \delta^{\prime} \eta{ }^{\prime} \kappa \omega \mu \dot{\varepsilon} \tau \alpha$

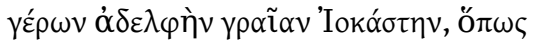

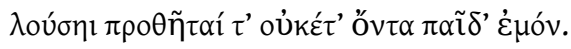

Ed io, vecchio, sono venuto da Giocasta, mia vecchia sorella, perché lavi ed esponga

il corpo del mio defunto figlio.

17 Stazio, al contrario, unifica le due sequenze, come abbiamo visto, e fa confliggere sentimenti di un padre con quelli di un potente, per mostrare come l'amore per il trono travolga ogni cosa.

Leggiamo le parole di Creonte (Theb. 12, 72-84):

"o nisi magnanimae nimius te laudis inisset ardor, Echionios mecum uenerande penates atque ultra recture puer, uenientia qui nunc gaudia et ingratum regni mihi munus acerbas! tu superum conuexa licet coetusque perenni (credo equidem) uirtute colas, mihi flebile semper numen eris; ponant aras excelsaque Thebae templa dicent: uni fas sit lugere parenti. et nunc heu quae digne tibi sollemnia quasue 
largiar exequias? nec si fatale potestas

Argos et impulsas cineri miscere Mycenas,

meque super, cui uita (nefas!) et sanguine nati

partus honos...

“Ah, figlio mio, se non t'avesse posseduto il desiderio ardente e smisurato di nobile gloria, tu degno d'onore avresti governato con me e dopo di me la città di Echione, $\mathrm{e}$ ora invece amareggi i successi che mi sono capitati e l'ingrato dono del trono! E anche se con la tua virtù imperitura ti sei conquistato il cielo e la compagnia degli dèi (lo credo davvero!), per me sarai sempre causa di pianto, per quanto tu sia un dio. Possa Tebe consacrarti altari e dedicarti alti templi: solo a tuo padre sia permesso di essere in lutto. E adesso, ahimè, quale cerimonia, quali esequie potrò offrirti degne del tuo valore? Non riuscirei mai a darti il tributo che meriti, neppure se avessi il potere di mescolare alle tue ceneri Argo funesta e Micene abbattuta, e se immolassi me stesso, io che debbo la mia vita e la gloria (orrore!) al sangue di mio figlio...

19 Solo pochi cenni sono dedicati al desiderio di gloria di Meneceo (vv. 72-3) e alla sua virtus, che lo rende tale da essere venerato come un dio (vv. 76-9) ${ }^{25}$ : il resto è incentrato su Creonte stesso, re-genitore, e non più vecchio inerme. Con il figlio avrebbe governato Tebe (vv. 73-4 mecum... recture) e a lui solo deve essere concesso di portare il lutto (v. 79): ma soprattutto, è il sangue di Meneceo che gli ha procurato il trono (vv. 83-4). La smania di protagonismo del tiranno fa sì che Creonte prefiguri in pochi versi la propria mancata carriera politica in un luogo che non vi è deputato.

Modello principale di questa scena è il lamento di Mezenzio sul cadavere di Lauso (Verg. Aen. 10, 846ss.) ${ }^{26}$. I due discorsi si aprono con una movenza affine e speculare, tantane me tenuit vivendi, nate, voluptas... 'fui preda di tanta brama di vivere, o figlio...' (Aen. 10, 846) magnanimae nimius te laudis inisset / ardor... (Theb. 12, 72-3); e sviluppano in parte punti comuni, che la riscrittura di Stazio, però, rovescia e perverte. In Aen. 10, 849 Mezenzio constata di essere in vita grazie alla morte del figlio (morte tua vivens? 'vivendo grazie alla tua morte?'); anche Creonte tocca questo tema, ma con un'aggiunta fondamentale $(12,83-4)$ : cui vita (nefas!) et sanguine nati / partus honos. Non solo la vita: il padre è debitore al figlio anche del regno, e anzi, forse questo ha valore maggiore agli occhi di Creonte, come induce a pensare il rilievo dato a partus honos, in enjambement seguito da cesura ${ }^{27}$.

Se la morte di Lauso riesce a umanizzare Mezenzio (che impara così dal dolore, come un eroe tragico $)^{28}$, Creonte ribadisce invece la sua natura tirannica, ed esacerba la propria sofferenza pensando al piacere che avrebbe provato dividendo il potere con Meneceo, lasciandolo poi unico sovrano (v. 74 ultra recture). Questi, con la sua morte, ha privato il padre di ogni gioia che gli sarebbe derivata dal regno, quello stesso regno che Creonte ha riconosciuto di dovere proprio al suicidio del figlio (12, 74-5): venientia qui nunc / gaudia et ingratum regni mihi munus acerbas; la gioia mancata di Creonte è qui accostata al munus regni e si identifica così con i piaceri del trono. Vengono in mente le parole di un altro padre sul cadavere del figlio, Evandro, che in Verg. Aen. 11, 180-1 chiede all'alleato Enea di vendicare l'uccisione di Pallante; una gioia che egli non cerca per la sua vita, ma per rallegrare un giorno il figlio morto con l'annuncio della vendetta: non vitae gaudia ${ }^{29}$ quaero, / nec fas, sed gnato manis perferre sub imos 'non chiedo questa gioia per la mia vita, / non è lecito, ma per recarla a mio figlio nel regno profondo dei morti'. Per Evandro, morto Pallante, i gaudia non sono più leciti (v. 181 nec fas); Creonte, invece, proprio all'inizio del compianto su Meneceo, pensa alla gioia che la scomparsa del figlio gli ha guastato (v. 75 acerbas): il possesso del trono tebano ${ }^{30}$. Tutti gli affetti del nuovo sovrano 
sembrano dunque contrarsi nello stretto orizzonte del regnum, che diviene così il suo unico paradigma assiologico.

In questa sezione, dunque, il confronto con l'Eneide fa emergere un aspetto importante del discorso politico portato avanti nella Tebaide: sovrano assoluto di Tebe, Creonte (come già Edipo) diventa l'immagine di una dicotomia tra le categorie di 're' e di 'genitore', che fa sì che neppure piangendo il figlio morto egli possa dimenticare la propria carica regale. E c'è forse di più. Se tradizionalmente il buon governante è elogiato come padre del suo popolo e della sua patria - un concetto che la cultura romana aveva sviluppato anche nella categoria encomiastica del pater patriae $e^{31}$ - Stazio, dopo aver presentato Creonte come un genitore capace di anteporre all'amore per il figlio quello per il trono, mette in discussione anche la bontà di un simile padre nei confronti del suo popolo. Il re di Tebe va così incontro a un duplice fallimento, come padre biologico e come padre 'politico': la conferma narrativa si avrà quando il tiranno, incurante dello stato di prostrazione del suo popolo uscito dalla guerra fratricida, lo costringerà a prendere nuovamente le armi contro Teseo $(12,698-709)^{32}$.

Ma torniamo al monologo di Creonte, con cui Stazio delinea il ritratto del tiranno. Nella seconda metà del suo lamento, il re confronta la propria condizione con quella di Edipo; entrambi sono padri che una guerra senza senso ha privato dei figli (Theb. 12, 84-92):

eademne dies, eadem impia bella

te, puer, et diros misere in Tartara fratres?

et nunc Oedipodi par est fortuna doloris

ac mihi? quam similes gemimus, bone Iuppiter, umbras!

accipe, nate, tui noua libamenta triumphi,

accipe et hoc regimen dextrae frontisque superbae

uincula, quae patri minimum laetanda dedisti.

regem te, regem tristes Eteocleos umbrae

aspiciant.

... Davvero furono lo stesso giorno e la stessa empia guerra a portare via te, ragazzo, e a inviare al Tartaro i maledetti fratelli? E ora la mia sorte, nel dolore, è pari a quella di Edipo? Ah, come si somigliano, mio buon Giove, le ombre per cui versiamo il nostro pianto! Ricevi nuove offerte per il tuo trionfo, figlio mio, e accetta anche lo scettro della mia destra e il diadema che cinge la superba fronte, quello che tu stesso hai donato al padre perché non ne traesse alcuna gioia. $\mathrm{E}$ che la mesta ombra di Eteocle possa vederti re, sì re".

Le parole di Creonte suonano cariche di sarcasmo sdegnato: egli si chiede se ora la sua condizione di padre privato del figlio lo renda davvero pari al suo nemico Edipo (v. 86 et nunc Oedipodi par est fortuna dolori / ac mihi?), e le umbrae di Eteocle, Polinice e Meneceo sono con ironia definite similes. Dopo la morte, i tre dovrebbero trovarsi in una condizione di uguaglianza, ma Creonte sembra sovvertire anche questa legge di natura, suggerendo che essi, in realtà, non sono simili ${ }^{33}$ : i figli di Edipo sono chiamati diros... fratres (85), in forte contrasto morale con il semplice puer con cui egli si rivolge al figlio nello stesso verso. Opportunamente, la critica ha individuato per questa scena un modello omerico ${ }^{34}$, l'incontro tra Priamo e Achille nell'ultimo libro dell'Iliade. Per riscattare il corpo di Ettore, Priamo osa penetrare nell'accampamento nemico e porsi di fronte all'uccisore del figlio: la sofferenza ha impartito ai due una lezione amara e universale, quella della comunanza degli uomini di fronte al dolore. Entrambi piangono pensando ai propri cari, Ettore, Peleo, Patroclo (Hom. Il. 24, 507-12):

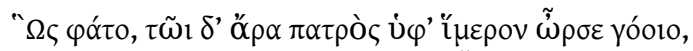

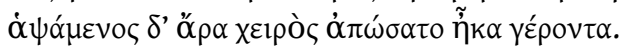

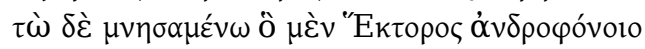




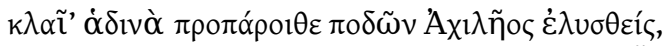

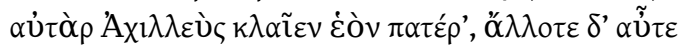

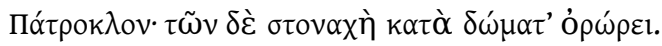

Così disse, e suscitò nell'altro il desiderio di pianto / per suo padre. Prese la mano del vecchio e la scostò dolcemente: / entrambi ricordavano, l'uno Ettore sterminatore, / e piangeva fitto, rannicchiato ai piedi di Achille; / Achille piangeva fitto quando suo padre e quando Patroclo, il loro pianto si levava attraverso le stanze.

Ma la ferocia di Creonte, si è visto, rovescia ogni tentativo di comunanza in nome del dolore. Il lettore che ha in mente l'episodio con cui si chiude l'Iliade si potrebbe forse attendere, a questo punto, una riproposizione del modello omerico - che c'è, ma viene cambiato di segno: 'in Thebaid 12, however, the effect of this line of thinking is opposite, as Creon uses it sarcastically', commenta K. Pollmann ${ }^{35}$. La carica ironica delle domande e delle esclamazioni aggrava l'ira del tiranno; la consapevolezza della condivisa sofferenza umana poteva placare la crudeltà di Achille nei confronti del nemico, ma non quella di Creonte. Come per fugare ogni dubbio in proposito, egli si rivolge al figlio menzionandone il trionfo (v. 88 tui... triumphi), assegnandogli lo scettro e la corona di Tebe (vv. 89-90) ${ }^{36}$, così che l'ombra di Eteocle, mesta e sconfitta, lo veda re (vv. 91-2 regem te, regem tristes Eteocleos umbrae / aspiciant).

La ferocia di Creonte va oltre: dopo aver quasi incoronato l'ombra del figlio, il nuovo sovrano di Tebe pensa alla pena da riservare ai corpi dei nemici (12, 92-103):

Simul haec dicens crinemque manumque

destruit, accensaque iterat uiolentius ira:

"saeuum agedum inmitemque uocent si funera Lernae

tecum ardere ueto; longos utinam addere sensus

corporibus caeloque animas Ereboque nocentes

pellere fas, ipsumque feras, ipsum unca uolucrum

ora sequi atque artus regum monstrare nefandos!

ei mihi, quod positos humus alma diesque resoluet!

quare iterum repetens iterumque edico: suprema

ne quis ope et flammis ausit iuuisse Pelasgos;

aut nece facta luet numeroque explebit adempta

corpora; per superos magnumque Menoecea iuro."

E così dicendo, si spogliò la chioma e la mano, e riprese a dire ancor più veemente con ira che gli bruciava il cuore: "Mi chiamino pure crudele e inumano se vieto di ardere insieme a te i morti di Argo; magari potessi infliggere a quei corpi lunghe sofferenze, scacciando quell'anime colpevoli dal cielo e dall'Erebo, e potessi io stesso inseguire le bestie feroci e i becchi adunchi degli uccelli e mostrare loro le maledette membra di quei re! Purtroppo la terra nutrice e il sole dissolveranno (ahimè!) i loro corpi là dove sono distesi! Perciò lo ripeto ancora e ancora pronunzio questo editto: che nessuno osi soccorrere i Greci con l'estremo tributo d'un rogo; altrimenti pagherà con la morte il suo gesto e andrà a completare il numero dei defunti. Lo giuro sugli dèi e sul grande Meneceo".

Alle formule del rito funebre ${ }^{37}$ Creonte intreccia scoppi d'ira e giuramenti di vendetta: la pietas universale, che Achille dimostrava di aver appreso dal dolore di Priamo, qui è vanificata del tutto. E sono ancora una volta precisi riferimenti intertestuali - ora lucanei - a dimostrarlo.

Come tutti gli epici flavi, Stazio si confronta con il modello di Virgilio, ma lo fa attraverso la lezione dell'epica e del teatro di età neroniana. L'influenza di Lucano sulla Tebaide è un tema vastissimo ${ }^{38}$ : qui basterà ricordare che la 'memoria diffusa' ${ }^{39} \mathrm{di}$ numerosi luoghi del Bellum civile diventa, nel poema di Stazio, la chiave per 
approfondire il discorso sul potere assoluto e sulle perversioni che esso comporta - il cuore tematico della Tebaide. Anche in questa sequenza.

Il lamento funebre di Creonte dà una prova ulteriore della natura tirannica del personaggio, come mostra il divieto di seppellire i corpi. Il dettaglio faceva parte della tradizione mitica e della vicenda già messa in scena da Sofocle ${ }^{40}$ : ma il sadismo con cui Creonte impartisce gli ordini rimanda più al Cesare di Lucano sul campo di Farsalo che alla tragedia attica.

Come ha messo in luce Helen Lovatt ${ }^{41}$, è attraverso il filtro lucaneo che va letto il finale della Tebaide - almeno, questa parte del finale. Il delirio di onnipotenza che spinge Cesare in Luc. 7, 797-803 a proibire i funerali dei caduti riecheggia nella folle chiusa del lamento funebre di Creonte ${ }^{42}$. Il divieto dell'Antigone sofoclea potrà essere empio o contro natura, ma segue (in maniera problematica) la logica di un governante che si trovi a prendere il potere dopo una guerra civile; lo stesso divieto, nella Tebaide, è invece espressione dell'ira di un tiranno (Theb. 12, 92-3 simul haec dicens crinemque manumque / destruit, accensaque iterat violentius ira; da confrontare con l'ira che detta il comportamento di Cesare in Luc. 7, 809-10 nil agis hac ira: tabesne cadavera solvat / an rogus, haud refert... 'con quest'ira non ottieni nulla: che dissolva i corpi la putredine o il fuoco, non fa differenza...').

31 La memoria lucanea garantisce in questo modo il rovesciamento dell'intertesto omerico: terminando il monologo con l'espressione della sua rabbia, Creonte conferma la propria natura ferina, accostandosi semmai all'Achille furibondo che crede di poter placare il suo dolore per la morte di Patroclo solo lasciando ai cani il corpo di Ettore (Hom. Il. 23, 19-26).

32 È la formulazione stessa dell'editto che sottolinea la ferinità del tiranno Creonte.

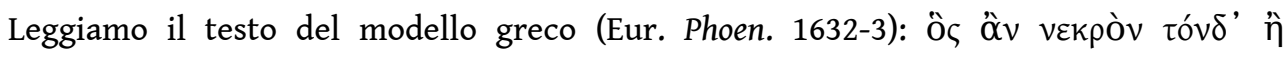

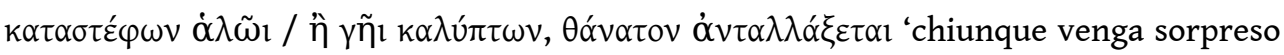
a incoronare o a coprire di terra questo cadavere, sarà punito con la morte'. Nessuna animosità, nessuno scatto d'ira: ben diverse suonano le parole di Creonte in Theb. 12, 94-9: saevum agedum inmitemque vocent si funera Lernae / tecum ardere veto; longos utinam addere sensus / corporibus caeloque animas Ereboque nocentes / pellere fas, ipsumque feras, ipsum unca volucrum / ora sequi atque artus regum monstrare nefandos! / Ei mihi, quod positos humus alma diesque resolvet!

L'ira spinge Creonte al delirio: egli desidera torturare i cadaveri dei caduti, tenere lontane le loro anime dal cielo e dall'inferno ${ }^{43}$; addirittura, in un accesso di sadismo degno di un tiranno senecano, vorrebbe che i corpi riacquistassero sensibilità per sentire ancora di più il dolore dei tormenti. Sono soprattutto i vv. 97-8 a mostrare la metamorfosi ferina di Creonte, che vorrebbe seguire le bestie feroci, indicando loro $\mathrm{i}$ cadaveri di cui fare scempio ${ }^{44}$; un eccesso a cui né Achille, né il Creonte 'tragico' arrivavano. Ma qui Stazio non si limita a raffigurare il tiranno della tradizione, va oltre, recuperando la memoria di un personaggio lucaneo: Eritto. La ferocia della maga la portava a unirsi a fiere e rapaci nello sconciare i cadaveri insepolti, e a gareggiare con loro in efferatezza (Luc. 6, 550-3):

Et quodcumque iacet nuda tellure cadaver, ante feras volucresque sedet nec carpere membra vult ferro manibusque suis morsusque luporum expectat siccis raptura e faucibus artus. 
Siede presso i cadaveri che giacciono sulla nuda terra, precedendo le belve e i rapaci; e non preferisce dilaniare le membra con il ferro o con le mani, ma aspetta che le mordano i lupi per strapparle dalle loro gole fameliche.

I desideri di Creonte sono così paragonati ai gesti concreti di Eritto ${ }^{45}$, ma con una precisazione 'politica': le membra che il tiranno vuole veder sbranate sono quelle dei re (v. 98) suoi predecessori, coloro ai quali egli imputa la morte di Meneceo.

Il comportamento di Creonte, insomma, è quello di una fiera ${ }^{46}$. I suoi deliri sembrano concretizzare la metafora di Platone, che in Rep. 565d-566a aveva teorizzato la bestialità del tiranno, e insieme confermare quanto Seneca aveva sostenuto sulla crudeltà del potente. Nel De clementia il passaggio da uomo a bestia è espresso con chiarezza: crudelitas minime humanum malum est indignumque tam miti animo; ferina ista rabies est, sanguine gaudere ac vulneribus et abiecto homine in silvestre animal transire "la crudeltà è un male per nulla umano e indegno di un così mite animo; è un furore bestiale gioire alla vista del sangue e delle piaghe e, gettata via la propria umanità, trasformarsi in una fiera dei boschi' $(1,25,1)^{47}$. E l'ossessione di Creonte, quella di accompagnare le belve a divorare i cadaveri, sembra riecheggiare ancora le ben note riflessioni di Seneca sul comportamento sanguinario del tiranno (in questo caso Alessandro): quid enim interest, oro te, Alexander, leoni Lysimachum obicias an ipse laceres dentibus tuis? tuum illud os est, tua illa feritas. O quam cuperes tibi potius ungues esse, tibi rictum illum edendorum hominum capacem! 'Alessandro, che differenza c'è, ti chiedo, se getti Lisimaco in pasto a un leone o se lo dilani tu stesso con i tuoi denti? tue sono quelle fauci, tua quella ferocia. Quanto avresti desiderato averli piuttosto tu, quegli artigli, quelle fauci grandi da poter divorare gli uomini!' (ibid.). Quel che in Seneca (e nella tradizione sulla tirannide) era una similitudine moraleggiante, per la maga Eritto è diventato una concreta consuetudine; per Creonte, terzo vertice del triangolo, è un desiderio che rivela con toni lucanei la natura ferina del tiranno. stata scalzata via dall'empietà di Cesare e dalla ferocia di Eritto.

Creonte, però, non è l'unico tiranno presente nel finale della Tebaide: per farne emergere i tratti peculiari, Stazio chiede al lettore di confrontarlo con Edipo. Il dialogo tra i due (Theb. 11, 677-707) mette a nudo la loro comune appartenenza a un orizzonte assiologico che non riesce - e forse non può - uscire dall'ossessione del potere tirannico. Dopo che Creonte ha scacciato Edipo da Tebe, quest'ultimo esplode in una tirata in cui alterna maledizioni e suppliche in maniera confusa e quasi delirante: augura a Creonte di subire la sorte che egli stesso, re di Tebe, ha dovuto subire, per poi concludere con la richiesta al rex magnus di una guida (v. 707).

Già Paola Venini notava la grande differenza tra questa scena e il suo modello euripideo: in Phoen. 1595ss., Edipo mira solo a impietosire Creonte, implorandolo di non condannarlo all'esilio ${ }^{48}$. Nella Tebaide il nuovo re di Tebe è già diventato tiranno, e non recede dalla sua posizione: ma bisogna sottolineare che Edipo conserva ancora la propria natura autocratica e tiene testa a Creonte in versi da cui emerge l"estetica della tirannia' elaborata dal pensiero senecano, il gusto perverso per l'esercizio di una crudeltà senza misura.

In Euripide, Edipo supplica Creonte di non scacciarlo da Tebe - per lui sarebbe la morte (Eur. Phoen. 1620-1):

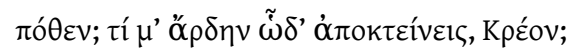

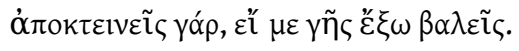


Dove? Perché mi uccidi così, Creonte? Mi ucciderai, infatti, se mi scaccerai dalla mia terra.

Stazio non lascia cadere questo spunto ${ }^{49}$, ma ne fa il vero manifesto di una sfida in saevitia tra tiranni. Ecco i versi (Theb. 11, 690-5):

... mihine ulla minaris

supplicia, aut ullos reris superesse timores?

Linquere tecta iubes? Caelum terramque reliqui

sponte, atque ultricem crudelis in ora retorsi

non ullo cogente manum; quid tale iubere,

rex inimice, potes?

Mi minacci qualche castigo o pensi forse che mi resti qualcosa che io possa temere?

Mi ordini dunque di lasciare la mia casa? Ma io ho già lasciato di mia volontà il cielo

e la terra, e, crudele, ho ritorto la mia mano vendicatrice contro i miei stessi occhi,

senza che nessuno mi costringesse: cosa puoi mai impormi tu di altrettanto

doloroso, o re, mio nemico?

40

versi è chiarissima: Creonte non può nuocere a Edipo senecano, Edipo si vanta di aver prevenuto e superato Creonte stesso: l'esilio che gli è senecano, Edipo si vanta di aver prevenuto e superato Creonte stesso: l'esilio che gli è minacciato è nulla, in confronto a quello cui proprio lui si è condannato spontaneamente (vv. 692-3 caelum terramque reliqui / sponte), accecandosi e rinchiudendosi nei recessi della reggia tebana. La sua mano, senza che nessuno lo obbligasse, ha fatto giustizia ${ }^{50}$; Edipo ha sconfitto Creonte sul suo stesso terreno, la crudeltà incomparabile del castigo.

'E non sia pena che la sua somigli': castigandosi senza alcuna misura, Edipo si è messo al riparo da Creonte. Come Tieste nell'ottica di Atreo, Edipo è già una punizione per se stesso: et quid esse tam saevum potest / quod superet illum? 'e cosa ci può essere di tanto crudele da superare lui?' (Sen. Thy. 196-7). Non certo l'esilio, tanto temuto nelle Fenicie euripidee. Il re patricida e incestuoso è il punitore di se stesso, la sua coscienza è un castigo a cui Creonte non può arrivare ${ }^{51}$ : 'quem, genitor, fugis?' 'da chi fuggi, padre?'chiede Antigone al padre nelle Phoenissae di Seneca. La risposta di Edipo possiede una lucidità e una profondità psicologica senza precedenti: 'me fugio, fugio conscium scelerum omnium / pectus, manumque hanc fugio et hoc caelum et deos / et dira fugio scelera quae feci innocens' 'fuggo da me stesso, fuggo dal mio animo complice di tutti i miei delitti, fuggo da questa mano, da questo cielo e dagli dèi, e fuggo dai delitti orribili che ho commesso innocente' (vv. 215-7).

Il lamento di Edipo sul cadavere dei figli ${ }^{52}$ segna un punto di svolta fondamentale nell'economia del poema: una metamorfosi che il dibattito critico ha giudicato di volta in volta psicologicamente credibile o posticcia, interrogandosi sulle sue cause e sull'efficacia della sua rappresentazione ${ }^{53}$. È difficile prendere una posizione netta; nel finale del poema Edipo è profondamente mutato rispetto al primo libro, eppure conserva ancora quegli stessi tratti da tiranno che all'inizio della vicenda lo avevano portato a maledire i figli. L'intreccio di padre e autocrate - un nesso violentemente ossimorico nel mondo della Tebaide - è inscindibile, come testimonia anche la parabola di Creonte. "Generis consortia ferro / dissiliant" "che il ferro faccia a pezzi i vincoli del sangue', aveva chiesto Edipo a Tisifone (1,84-5); al termine del poema, questa preghiera per la dissoluzione dei legami familiari rivela tutta la sua portata distruttiva, estendendosi all'intera famiglia di Edipo: anche il legame tra padri e figli ${ }^{54}$ è destinato a soccombere all'amore per il trono. 


\section{BIBLIOGRAFIA}

AMBÜHL 2015: A. Ambühl, Krieg und Bürgerkrieg bei Lucan und in der Griechischen Literatur. Studien zur Rezeption der Attischen Tragödie und der Hellenistischen Dichtung im 'Bellum Civile', Berlin-München-Boston 2015.

ARIEMMA 2008: E. Ariemma, Odia fraterna, fraternae acies: i gemelli gladiatori in Silio Italico (Pun. 16, 527-48), «Lexis» 26, 2008, pp. 325-69.

AUGOUSTAKIS 2016: A. Augoustakis, Burial and Lament in Flavian Epic: Mothers, Fathers, Children, in N. Manioti (ed.), Family in Flavian Epic, Leiden-Boston 2016, pp. 276-300.

BERNO 2016: F. R. Berno, Lo scettro cruento di Edipo. Sen. Oed. 642 e dintorni, «Pan» 5, 2016, pp. 61-73.

BESSONE 2011: F. Bessone, La Tebaide di Stazio. Epica e potere, Pisa-Roma 2011.

BESSONE cds.: F. Bessone, Allusive (Im-)pertinence in Statius'Epic, in D. Nelis (eds.), Intertextuality in Flavian Poetry, Berlin-Boston, cds.

BOYLE 2011: Seneca, Oedipus, ed. with Intr., Transl., and Comm. by A. J. Boyle, Oxford 2011.

BRAUND 2009: Seneca, De Clementia, ed. by S. Braund, Oxford 2009.

BRIGUGLIO 2017: S. Briguglio, Fraternas acies. Saggio di commento a Stazio, Tebaide, 1, 1-389, Alessandria 2017.

BRIGUGLIO 2017a: S. Briguglio, 'O voluttà del soglio!'. Eros e potere nella Tebaide di Stazio in Atti del III Seminario CUSL, «Biblioteca di ClassicoContemporaneo III» 5, 2017, pp. 310-26.

DAVIS 1994: P. J. Davis, The Fabric of History in Statius'Thebaid, in C. Deroux (ed.), Studies in Latin Literature and Roman History VII, Bruxelles 1994, pp. 464-83.

DELARUE 2000: F. Delarue, Stace, poète épique. Originalité et cohérence, Louvain-Paris 2000.

DOMINIK 1990: W. J. Dominik, Monarchal Power and Imperial Politics in Statius' Thebaid, in A. J. Boyle (ed.), The Imperial Muse. Ramus Essays on Roman Literature of the Empire. Flavian Epicist to Claudian, Bendigo (Victoria) 1990, pp. 74-97.

DOMINIK 1994: W. J. Dominik, The Mythic Voice of Statius: Power and Politics in the Thebaid, Leiden-New York-Köln 1994.

FEENEY 1991: D. C. Feeney, The Gods in Epic. Poets and Critics of the Classical Tradition, Oxford 1991.

FRANCHET D'ESPÈREY 1999: S. Franchet d'Espèrey, Conflit, violence et non-violence dans la Thébaïde de Stace, Paris 1999.

FUCECCHI 2015: M. Fucecchi, Passato da rimuovere e passato da rivivere: l'incubo della guerra civile (e la sua 'metabolizzazione') nell'epica flavia, in P. Esposito, C. Walde (a c. di), Letture e lettori di Lucano. Atti del Convegno Internazionale di Studi, Fisciano 27-29 marzo 2012, Pisa 2015, pp. 231-53.

GANIBAN 2007: R. Ganiban, Statius and Virgil. The Thebaid and the Reintrepretation of the Aeneid, Cambridge 2007.

GERVAIS 2015: K. Gervais, Parent-Child Conflict in the Thebaid, in W. J. Dominik, C. E. Newlands, K. Gervais (eds.), Brill's Companion to Statius, Leiden-Boston 2015, pp. 221-39. 
HARDIE 1993: P. Hardie, The Epic Successors of Virgil. A Study in the Dynamics of a Tradition, Cambridge 1993.

HARRISON 1991: Vergil. Aeneid 10, ed. by S. J. Harrison, Oxford 1991.

HORSFALL 2003: N. Horsfall (ed.), Virgil. Aeneid 11: A Commentary, Leiden-Boston 2003.

KEITH 2004-5: A. Keith, Ovid's Theban Narrative in Statius' Thebaid, «Hermathena» 177/8, 2004-5, pp. 181-207.

LAMARI 2010: A. A. Lamari, Narrative, Intertext, and Space in Euripides' ‘Phoenissae', Berlin-New York 2010.

LANZARONE 2016: M. Annaei Lucani Belli Civilis liber VII, a c. di N. Lanzarone, Firenze 2016.

LA PENNA 1979: A. La Penna, Atreo e Tieste sulle scene romane, in Id., Fra teatro, poesia e politica romana, Torino 1979, pp. 127-41.

LA PENNA 1980: A. La Penna, Mezenzio: una tragedia della tirannia e del titanismo antico, «Maia» 32, 1980, pp. 3-30.

LOVATT 1999: H. Lovatt, Competing Endings: Re-reading the End of the Thebaid through Lucan, «Ramus» 28, 1999, pp. 126-51.

MALASPINA 2005²: L. Annaei Senecae de clementia liberi duo, prolegomeni, testo critico e commento a c. di E. Malaspina, Alessandria $2005^{2}$.

MASTRONARDE 1994: Euripides, Phoenissae, ed. with Intr. and Comm. by D. J. Mastronarde, Cambridge 1994.

MICOZZI 1999: L. Micozzi, Aspetti dell'influenza di Lucano nella “Tebaide”, in P. Esposito, L. Nicastri (a c. di), Interpretare Lucano, Napoli 1999, pp. 343-87.

MICOZZI 2002: L. Micozzi, Il tema dell'addio: ripetizione, sperimentalismo, strategie di continuità e altri aspetti della tecnica poetica di Stazio, «Maia» 54, 2002, pp. 51-70.

MICOZZI 2004: L. Micozzi, Memoria diffusa di luoghi lucanei nella Tebaide di Stazio, in P. Esposito, E. M. Ariemma (a c. di), Lucano e la tradizione dell'epica latina. Atti del convegno internazionale di studi, Fisciano-Salerno, 19-20 ottobre 2001, Napoli 2004, pp. 137-51.

NISBET-HUBBARD 1970: A Commentary on Horace, Odes, Book I, ed. by. R. G. M. Nisbet and M. Hubbard, Oxford 1970.

OGILVIE 1965: A Commentary on Livy. Books 1- 5, by R. M. Ogilvie, Oxford 1965.

POLLMANN 2004: K. F. L. Pollmann, Statius, Thebaid 12. Introduction, Text, and Commentary, Paderborn-München-Wien-Zürich 2004.

RIPOLL 1998: F. Ripoll, La morale héroïque dans les épopées latines d'époque flavienne: tradition et innovation, Louvain-Paris 1998.

ROBINSON 2011: A Commentary on Ovid's Fasti Book 2, ed. by M. Robinson, Oxford 2011.

ROCHE 2015: P. Roche, Lucan's De bello civili in the Thebaid, in W. J. Dominik, C. E. Newlands, K. Gervais (eds.), Brill's Companion to Statius, Leiden-Boston 2015, pp. 393-407.

ROSATI 2006: Libido amandi e libido regnandi, ovvero elegia e potere nel teatro senecano, «Dioniso» 5, 2006, pp. 94-105.

SCHIESARO 2003: A. Schiesaro, The Passions in Play. Thyestes and the Dynamics of Senecan Drama, Cambridge 2003. 
TARRANT 1985: Seneca's Thyestes, ed. by R. J. Tarrant, Atlanta 1985.

VENINI 1970: P. Papini Stati Thebaidos liber XI, a c. di P. Venini, Firenze 1970.

VENINI 1971: P. Venini, Studi staziani, Pavia 1971.

\section{NOTE}

1. Cf. ad es. Dominik 1990; 1994; Delarue 2000, p. 259; Ganiban 2007, p. 199; 205; sulla 'consapevolezza tragica' che grava sui personaggi della Tebaide, cf. ad es. Ripoll 1998, pp. 35ss.

2. Cf. ad es. Davis 1994; Keith 2004-5.

3. Bessone 2011, pp. 18ss.

4. Bessone 2011, p. 56: “C'è il senso di una ripartenza: Edipo ritratta la maledizione iniziale contro Eteocle e Polinice, e scaglia una nuova maledizione contro Creonte; rovescia, di fronte ai corpi dei figli, le parole usate per maledirli, e le ripete per maledirne il successore".

5. Le traduzioni dalla Tebaide sono di L. Micozzi (Milano 2011) e quella dall'Iliade di G. Paduano (Torino 1997). Le restanti sono di chi scrive.

6. Anche la menzione del regimen... cruentum rimanda una volta di più all'inizio del poema: cf. Edipo a Tisifone in Theb. 1, 83-4 indue quod madidum tabo diadema cruentis / unguibus abripui... 'cingi il diadema che, intriso di corruzione, io stesso mi sono strappato con le unghie insanguinate...'. In Stazio il motivo dei segni del potere insanguinati deriva in prima battuta da Seneca tragico (cf. Venini 1970 ad Theb. 11, 659), ma ha radici e propaggini ben più estese: cf. Berno 2016; Briguglio 2017 ad loc.

7. Cf. Gibson 2015; Briguglio 2017 ad loc.

8. Cf. anche Edipo in Theb. 11, 695-6 fugio excedoque nefandis / sedibus... 'ebbene fuggo, abbandono l'abominevole dimora...'.

9. Cf. Bessone 2011, p. 93; 150-1; Ariemma 2008. Il duello tra Eteocle e Polinice dovrebbe essere un monito ai posteri perché una simile guerra non si ripeta più: documentum compariva già nella descrizione dello scontro tra Corbis e Orsua, fratelli che si contendono il potere sulla loro città in Liv. 28, 21, 9 insigne spectaculum exercitui praebere documentumque quantum cupiditas imperii malum inter mortales esset 'offrirono all'esercito una notevole dimostrazione e una testimonianza di che grande sciagura fosse per gli uomini la brama di potere'.

10. Il 'ritorno al passato' è del resto l'immagine che meglio rappresenta la ciclicità del tempo a Tebe e la ricorsività nelle colpe; Edipo stesso, commettendo incesto con Giocasta, è ritornato, contro natura, alle sue origini: cf. Theb. 1, 234-5 ... inmeritae gremium incestare parentis / appetiit (monstrum!) proprios revolutus in ortus '... e [ha desiderato] macchiare con l'incesto il grembo della madre incolpevole, ritornando (cosa mostruosa!) alle origini della sua stessa vita', con Briguglio 2017 ad loc. per il precedente dell'Oedipus senecano.

11. Bessone 2011, p. 153.

12. Il punto di partenza per la caratterizzazione del tiranno a Roma resta La Penna 1979.

13. Fondamentale Rosati 2006.

14. Cf. Bessone cds.; Briguglio 2017a.

15. Blandus è uno dei termini del lessico erotico (almeno fin da Lucr. 1, 19) non per caso frequenti nella Tebaide quando viene descritta la passione del tiranno per il trono.

16. Cf. anche Theb. 1, 127-8 regendi / saevus amor 'la spietata bramosia del potere'. Il nesso amor regni è già in Liv. 40, 8, 18; ad esso si può in parte accostare anche la variante regni cupido, la brama di potere che scatena la lotta tra Romolo e Remo in 1, 6, 4 (loci paralleli in Ogilvie 1965 ad loc.) e che in Tac. Ann. 12, 47, 5 spinge Farasmane a macchiarsi del sangue dei propri congiunti; anche il Cesare di Lucano sul campo di Farsalo è flagrans cupidine regni 'ardente per la brama di potere' (Luc. 7, 240 con Lanzarone 2016). 
17. Cf. ancora Briguglio 2017a.

18. Impensus patriae paulo ante Menoeceus; per l'uso di impendo, cf. Venini 1970 ad loc.

19. Augoustakis 2016, pp. 288-9, confronta i lamenti di Creonte, Edipo e Licurgo sul corpo dei loro figli, evidenziando l'intreccio di paternità e regalità.

20. Cf. anche Augoustakis 2016, pp. 293-4.

21. Accetto (con Klotz, Lesueur, Hill, Shackleton Bailey e Hall) la lez. potes di P, e intendo così Creonte come soggetto di incipit; la maggioranza degli altri codici ha potest, difeso da Venini 1970. 22. Cf. Venini 1970 ad 11, 660.

23. Cf. Ganiban 2007, p. 199: "though Creon has been mourning the death of his son Menoeceus, such feelings of pietas are quickly subsumed by his desire for rule"; cf. anche p. 207, n. 1.

24. Su tutta la scena del lamento di Creonte (vv. 1308-34) grava il sospetto di guasti testuali: cf. Mastronarde 1994, pp. 512 ss.; Lamari 2010, p. 102.

25. Ripoll 1998, pp. 219-20, discute la posizione presa da Creonte di fronte all'eccessivo desiderio di gloria di Meneceo.

26. Cf. Bessone 2011, p. 151.

27. Al contrario, Mezenzio constata amaramente di aver macchiato il nome del figlio e di essere stato allontanato dal potere per le sue colpe (10, 851-2): idem ego, nate, tuum maculavi crimine nomen, / pulsus ob invidiam solio sceptrisque paternis 'io, figlio, ho macchiato il tuo nome con i miei delitti, io, scacciato con odio dal soglio e dal regno paterno'.

28. Cf. La Penna 1980; Harrison 1991 ad Verg. Aen. 10, 689-768.

29. Alcuni commentatori interpretano vitae come un dativo (persuasivamente: cf. EV, s.v. gaudium), altri come genitivo (cf. ThLL VI.2, 1717.70): per la questione cf. Horsfall 2003 ad loc.

30. Evandro, inoltre, constata la perversione dell'ordine naturale nella sopravvivenza di un genitore al figlio (Aen. 11, 160-1 contra ego vivendo vici mea fata, superstes / restaret ut genitor 'io, invece, rimanendo in vita ho vinto i miei fati, perché restassi genitore superstite'); e così Mezenzio (10, 848 tuane haec genitor per vulnera servor...? 'io, tuo padre, sono salvo grazie a queste tue ferite...?'). I due padri nei loro lamenti funebri deprecano di non essere morti al posto dei figli (Aen. 10, 854 omnis per mortis animam sontem ipse dedissem! 'e avessi dato la mia vita colpevole in mille morti!', con Harrison 1991 ad loc.; 11, 162-3 animam ipse dedissem / atque haec pompa domum me, non Pallanta, referret! 'io stesso avrei dato la vita, e questo corteo avrebbe riportato indietro me, non Pallante!'). Creonte, rex genitor, non insiste, invece, su questa eccezione perversa.

31. Cf. ad es. Cic. Rep. 1, 64; per una discussione del concetto nel suo sviluppo storico (che culmina con Plin. Pan. 2, 3), cf. Nisbet-Hubbard 1970 ad Hor. Carm. 1, 2, 70; Robinson 2011 ad Ov. Fast. 2, 127, Braund 2009 ad Sen. Clem. 1, 14, 2, con bibliografia.

32. Su questo passo, in cui viene condensata e rovesciata la scena tradizionale dei preparativi per la guerra, cf. Micozzi 2002; Bessone 2011, pp. 183.

33. Cf. Pollmann 2004 ad 84-7.

34. Cf. Pollmann 2004, p. 110-1.

35. Cf. Pollmann 2004, ibid.

36. Dove è significativa la movenza quasi celebrativa suggerita dall'anafora colonnare incipitaria di accipe: di pari efficacia è la geminatio al v. 91 di regem, quasi icastica dello smacco inflitto all'ombra di Eteocle.

37. Analizzate in Pollmann 2004 ad loc.

38. Cf. Venini 1971, pp. 45-54; 55-67; 68-79; Micozzi 1999; 2004; Roche 2015.

39. Cf. Micozzi 2004.

40. Diversamente, nelle Fenicie di Euripide, Creonte si limita a eseguire gli ordini di Eteocle: cf. vv. 774-7; 1646-72, con Mastronarde 1994 ad loc.

41. Cf. Lovatt 1999, spec. pp. 133-5, sulla 'secondarietà' di Creonte rispetto a Cesare; ma cf. anche Bessone 2011, pp. 151-2. 
42. Cf. Pollmann 2004, p. 112; Lanzarone 2016 ad 797-824. Ampia discussione delle fonti di Lucano e confronto con Stazio in in Ambühl 2015, pp. 259ss.; cf. pp. 262-3: "In der Tat zeichnet Statius seinen Creon als einen unmenschlichen Super-Tyrannen, der Lucans Caesar bei weitem überbietet".

43. Un'immagine senecana: cf. Oed. 658 con Boyle 2011 ad loc.

44. Sulla ferinità degli antieroi staziani cf. le osservazioni di Franchet d'Espèrey 1999, pp. 172-3; più in generale, sulla disumanizzazione nel poema, cf. pp. 190-7.

45. Le azioni di Eritto saranno di nuovo evocate indirettamente dalle parole di Argia, che chiede di sapere dove si trovi il corpo di Eteocle: cf. Theb. 12, 341-3: qua parte, precor, iacet ille nefandus / praedator? vincam volucres (sit adire potestas) / excludamque feras 'e da quale parte, dimmi, ti prego, giace quell'abominevole predatore? Supererò gli uccelli (se soltanto potessi avvicinarmi a lui) e scaccerò le bestie feroci' Luc. 6, 550-3 (supra); cf. Fucecchi 2015, p. 248 (ma per un cenno all'Eritto lucanea in questa scena cf. già Hardie 1993, p. 45).

46. Cf. anche Feeney 1991, p. 360.

47. Il confronto tra il tiranno e la fiera è già in Cic. Rep. 2, 48: cf. Malaspina $2005^{2}$ ad 1, 25, 1.

48. Cf. Venini 1970 ad 11, 677ss. Una differenza cruciale sta proprio nella rievocazione delle sventure passate: in Euripide esse sono solo un elemento patetico che rinforza la supplica, mentre l'Edipo di Stazio le richiama per augurarsi che si ritorcano contro Creonte, nuovo possessore del trono maledetto (cf. Theb. 11, 701-5 habeas Thebana regasque / moenia, quo Cadmus, quo Laius omine rexit / quoque ego; sic thalamos, sic pignora fida capessas, / nec tibi sit virtus fortunam evadere dextra, / sed lucem deprensus ames 'tieniti pure la città di Tebe e governala, con lo stesso destino con cui già governarono Cadmo e Laio, e con cui la governai io stesso. Possa tu avere figli e nozze cari, come io li ho avuti; ma senza il coraggio di sottrarti di tua mano al destino, continuando a restare attaccato alla vita, quando t'avrà afferrato').

49. Cf. Venini 1970 ad 11, 690s.

50. Cf. Theb. 1, 71ss., con Briguglio 2017.

51. Cf. anche Theb. 1, 49-54.

52. Cf. Theb. 11, 605-26. Una sezione importante in cui Stazio rielabora i versi del lamento di Teseo sul cadavere di Ippolito in Sen. Phaedr. 1114-7 o nimium potens, / quanto parentes sanguinis vinclo tenes, / Natura, quam te colimus inviti quoque. / Occidere volui noxium, amissum fleo 'o Natura, troppo potente! quanto è forte il legame di sangue con cui stringi i genitori! Quanto ti rispettiamo anche contro la nostra volontà! Ho voluto che il colpevole morisse, ma adesso che l'ho perso lo piango' 11, 607 vincis io miserum, vincis, Natura, parentem 'alla fine trionfi, o Natura! Sì, trionfi su questo povero padre' (cf. già Venini 1970 ad loc.; Franchet d'Espèrey 1999, pp. 305-6; Delarue 2000, p. 161). A sua volta, la maledizione di Teseo presenta qualche punto di contatto con la maledizione di Edipo nel primo libro: entrambi chiedono l'intervento divino contro i figli, riconoscendo la perversione delle proprie preghiere (Theb. 1, 59 adnue, Tisiphone, perversaque vota secunda 'rispondi benevola, o Tisifone... e asseconda i miei perversi desideri' Phaedr. 945 en perage donum triste, regnator freti! 'su, porta a compimento il tuo dono funesto, re del mare!'; 948 fer abominandam nunc opem gnato parens 'padre, concedi ora a tuo figlio un aiuto mostruoso'), entrambi hanno relazioni con il mondo infero, che è il luogo in cui metaforicamente dimora Edipo, capace di vedere, nella sua cecità, le creature di tenebra (Theb. 1, 46ss.), ed è anche il regno da cui è giunto incolume Teseo (Phaedr. 940 scis unde redeam, 'tu sai da dove faccio ritorno').

53. Cf. ad es. Feeney 1991, pp. 359-60; Franchet d'Espèrey 1999, pp. 297ss.; Ganiban 2007, pp. 195-7; Bessone 2011, p. 138, n. 3.

54. Sul tema cf. da ultimo Gervais 2015. 


\section{RIASSUNTI}

Questo articolo studia la metamorfosi di Creonte in tiranno nel finale della Tebaide: proprio mentre leva il suo lamento funebre sul corpo di Meneceo, egli rivela come in lui l'amore per il trono tebano prevalga addirittura su quello per il figlio. In questo senso Creonte perverte il modello virgiliano di due padri che piangono la morte dei figli, Evandro e Mezenzio, e si avvicina invece al suo antagonista, Edipo. Creonte, che vieta di seppellire i cadaveri dei caduti, richiama il modello di Cesare (ma anche di Eritto) in Lucano, e Edipo, che a suo dire si è inflitto una punizione tanto crudele che nemmeno il suo nemico può superare, esibisce significativi tratti dell' 'estetica della tirannia' delle tragedie senecane.

\section{INDICE}

Keywords : Stazio, Tebaide, Lucano, Seneca, tirannia, lamento funebre 